\title{
Embolización transcatéter con dispositivo, una promisoria opción en el tratamiento del secuestro pulmonar. Casos clínicos
}

\author{
ALEJANDRO ÁLVAREZ J. ${ }^{1}$, CAROL CLEVELAND P. ${ }^{2}$, KARINA GREEN M. ${ }^{2}$, \\ EUGENIO SANHUEZA H. ${ }^{3}$, JUNIA SILVA V. ${ }^{4}$
}

1. Pediatra, Cardiólogo Infantil, Servicio de Pediatría Unidad de Neonatología Hospital Guillermo Grant Benavente Concepción. Departamento de Pediatría, Facultad de Medicina, Universidad de Concepción.

2. Becada de Pediatría Beca Extendida APS, Servicio de Pediatría Hospital Las Higueras Talcahuano. Departamento de Pediatría Facultad de Medicina Universidad de Concepción.

3. Médico Anestesiólogo Cardiovascular, Unidad de Hemodinamia Hospital Las Higueras Talcahuano.

4. Pediatra, Broncopulmonar Infantil, Servicio de Pediatría Hospital Las Higueras Talcahuano.

Departamento de Pediatría, Facultad de Medicina, Universidad de Concepción.

\begin{abstract}
Transcatheter embolization device as a promising option in the treatment of pulmonary sequestration. Case reports

Introduction: Pulmonary sequestration (PS) has been treated for many years with conventional surgical removal of the ectopic lung tissue and ligation of aberrant vessels that arise from the aorta. There is evidence, especially in English-language literature that supports the use of transcatheter arterial embolization through a device for occlusion of the anomalous vessel, as a safe option and definitive treatment for intralobar PS. Objective: To show our experience pioneering the technique of PS treatment through transcatheter embolization. Case report: The case reports of two teenagers aged 13 and 14 and a 26-day-old newborn that developed intralobar PS are described. The diagnosis was made through computerized axial tomography (CT scan) and successfully and without complication, during early and long term follow-up, treated by arterial embolization transcatheter. Conclusion: The use of transcatheter arterial embolization is a less invasive and definitive treatment for patients with PS.
\end{abstract}

(Key words: Pulmonary sequestration, aberrant vessel, transcatheter therapy, intravascular embolization device).

Rev Chil Pediatr 2014; 85 (2): 197-202

\section{RESUMEN}

Introducción: El secuestro pulmonar (SP) ha sido tratado por muchos años con cirugía convencional efectuando remoción del tejido pulmonar ectópico y ligadura de los vasos sanguíneos aberrantes que nacen desde la aorta. Existe evidencia establecida, especialmente en literatura de habla inglesa que apoya el uso de la

Recibido el 7 de febrero de 2013, devuelto para corregir el 10 de junio de 2013, segunda versión 8 de agosto de 2013, tercera versión 17 de diciembre de 2013, aceptado para publicación el 27 de enero de 2014.

Este trabajo cumple con los requisitos sobre consentimiento /asentimiento informado, comité de ética, financiamiento, estudios animales y sobre la ausencia de conflictos de intereses según corresponda.

\section{Correspondencia a:}

Dr. Alejandro Álvarez Jara

E-mail: alalvare@udec.cl 
embolización arterial transcatéter mediante dispositivo para ocluir el vaso anómalo, como una opción de tratamiento seguro y definitivo para el SP intralobar. Objetivo: Mostrar nuestra experiencia pionera de la técnica de tratamiento del SP por medio de embolización transcatéter. Caso clínico: Presentamos los casos clínicos de dos adolescentes, de 13 y 14 años y de un recién nacido de 26 días de vida, portadores de SP intralobar, cuyos diagnósticos se realizaron a través de Tomografía Axial Computarizada (TAC) con contraste, tratados mediante embolización arterial transcatéter, con buen resultado y sin complicaciones en el seguimiento precoz y tardío. Conclusión: Con este tratamiento se logra en los pacientes afectados de SP resultados definitivos, menos invasivos y sin complicaciones al seguimento.

(Palabras clave: Secuestro pulmonar, vaso aberrante, tratamiento transcatéter, embolización con dispositivo intravascular).

Rev Chil Pediatr 2014; 85 (2): 197-202

\section{Introducción}

El secuestro pulmonar (SP) es un desorden genético que afecta al $0,15-1,7 \%$ de la población, habitualmente durante la niñez ${ }^{1,2}$. Se define como una masa quística de tejido pulmonar ectópico no funcionante sin comunicación con la vía aérea, irrigado por un vaso sanguíneo aberrante sistémico. Se clasifica anatómicamente en intralobar donde la masa se encuentra dentro del lóbulo pulmonar normal y carece de pleura propia y extralobar, cuando la lesión se ubica fuera del pulmón normal y tiene su propia pleura visceral. Los intralobares corresponden a más del $90 \%$ de los SP; $60 \%$ de estos se encuentran en los segmentos posterobasales del lóbulo inferior izquierdo ${ }^{2}$. Aproximadamente $98 \%$ ocurren en los lóbulos inferiores mientras que la afectación bilateral es rara. Los SP extralobares ocurren en el lado izquierdo en 95\% de los casos; de éstos, $75 \%$ se encuentran entre el hemidiafragma izquierdo y el lóbulo inferior izquierdo $\mathrm{o}^{2,3}$.

Los pacientes pueden permanecer asintomáticos durante años, realizándose el diagnóstico como un hallazgo a través de una radiografía de tórax. Cuando presentan síntomas, son inespecíficos e incluyen dolor torácico, hemoptisis, disnea e infecciones pulmonares recurrentes, entre otros. El tratamiento convencional es quirúrgico, realizando remoción del tejido y ligadura de los vasos sanguíneos aberrantes $^{2,3}$. Existe evidencia establecida, especialmente en la literatura pediátrica en los últimos años, que apoya el uso de la embolización arterial transcatéter mediante dispositivos intravasculares (coil, tapones, etc.), como un tratamiento, menos invasivo, seguro y de buen pronóstico para el SP intralobar ${ }^{1}$.

El objetivo de este reporte es mostrar nuestra experiencia pionera de la técnica de embolización transcatéter, ya que no existen publicaciones a nivel nacional del tratamiento percutáneo del SP.

\section{Caso 1}

Adolescente de 14 años de sexo femenino con historia de cuadros respiratorios recurrentes desde los 6 años de edad caracterizados por tos, broncorrea y sibilancias: en control en el policlínico de broncopulmonar, con los diagnósticos de daño pulmonar crónico secundario a neumonía asociada a ventilación mecánica durante el curso de una hemorragia cerebral interventricular en el período neonatal. No se cuenta con más antecedentes de dicho episodio.

Dentro del estudio etiológico de los cuadros respiratorios recurrentes destacaba espirometría, electrolitos del sudor e inmunoglobulinas, los cuales resultaron normales. La Tomografía Axial Computada (TAC) pulmonar sin contraste mostró la presencia de bronquiectasias. Por la persistencia de sintomatología se realizó nueva TAC con contraste que mostró extensas bronquiectasias en la base del hemitórax derecho, e imágenes sugerentes de SP del segmento póstero basal derecho (figura 1). También fue evaluada con ecocardiograma que resultó normal, se realizó un estudio angiográfico para confirmar el diagnóstico y para eventual embolización del SP.

Bajo sedación y anestesia local, se cateterizó arteria femoral derecha con introductor 6 French. Se efectuó angiografía en aorta as- 
cendente y descendente con catéter pig-tail 6 French, que mostró, a $2 \mathrm{~cm}$ sobre arteria renal, un vaso de diámetro máximo de $4 \mathrm{~mm}$ que ascendía en dirección al diafragma derecho. Este se canalizó con un catéter multipropósito de 4 French y se efectuó angiografía selectiva mostrando una red capilar arteriovenosa que drenaba en vena pulmonar derecha inferior y ésta en aurícula izquierda. Se realizó embolización del vaso anómalo con dispositivo de liberación controlado coil $\left(\mathrm{Cook}^{\mathrm{R}}\right)$ 5PDA5 ocluyendo el vaso en zona previa al nacimiento de la red capilar luego de comprobado que el dispositivo estaba estable se efectúa su liberación. A los 10 min post liberación se realizó una aortogra-

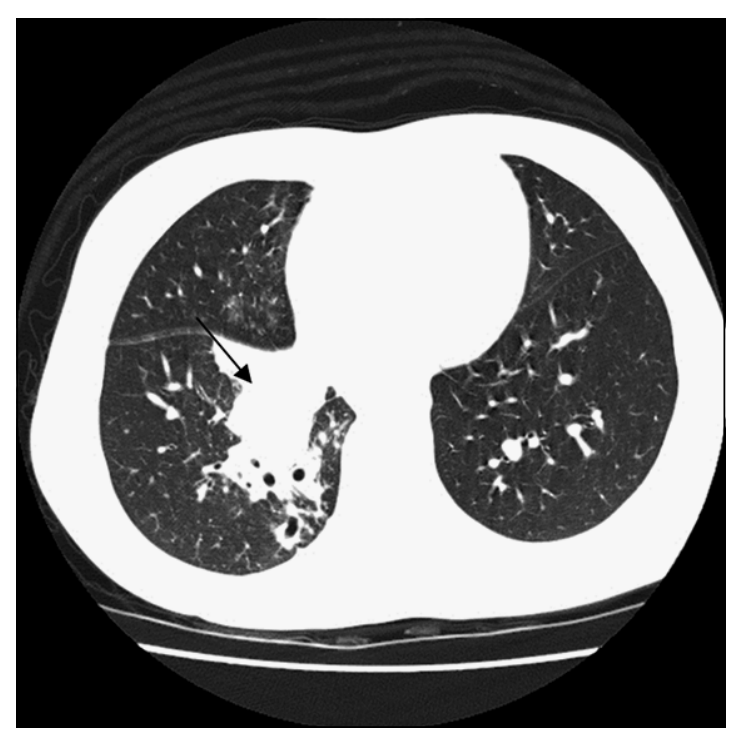

Figura 1. TAC pulmonar donde se visualiza imagen de consolidación intrapulmonar bien demarcada compatible con secuestro pulmonar intralobar. fía apreciándose la ausencia de flujo al vaso anómalo.

La paciente fue dada de alta al día siguiente previo control con radiografía tóracoabdominal que mostró dispositivo en sede (figura 2). Fue controlada a la semana, al mes, a los 6 meses, al año y dos años post procedimiento en el policlínico de broncopulmonar y cardiología infantil encontrándose en óptimas condiciones y asintomática.

\section{Caso 2}

Escolar de 13 años, sexo masculino en controles en el policlínico de broncopulmonar, con antecedentes de cuadros bronquiales obstructivos frecuentes e infecciones respiratorias recurrentes, con buena respuesta a tratamiento convencional.

Por referir dolor torácico se efectuó un TAC de tórax que pesquisó una imagen en el lóbulo superior derecho compatible con SP intralobar. Fue evaluado con electrocardiograma y ecocardiograma resultando normales. Se realizó estudio hemodinámico para lo cual se usó sedación y anestesia local con lidocaína, se cateterizó la arteria femoral derecha con introductor 5 French. Se efectuó una aortografía torácica con catéter Pig Tail 5 French donde se pesquisó, a nivel de la aorta torácica, un vaso aberrante de gran calibre que nacía de ésta y se dirigía al lóbulo superior del pulmón derecho con diámetro mínimo $2 \mathrm{~mm}$ y máximo $6 \mathrm{~mm}$ (figura 3 ).

Posteriormente, con catéter multipropósito 5 French se canalizó el vaso y posteriormente se procedió al cambio de introductor $\mathrm{N}^{\mathrm{o}} 6$ French y por medio de un catéter multipropósito se ubicó una guía de intercambio a distal

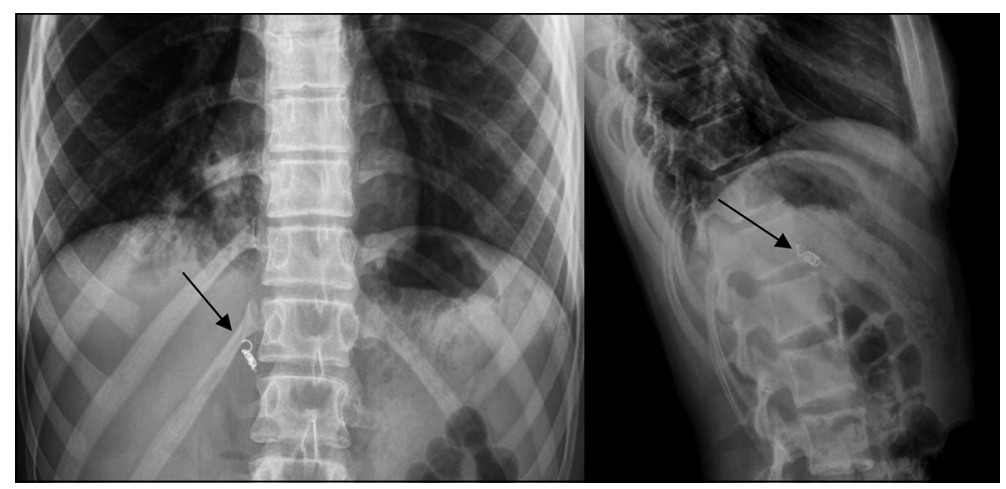

Figura 2. Radiografía toraco-abdominal donde se aprecia dispositivo coil $\left(\mathrm{Cook}^{\mathrm{R}}\right)$ en sede (flecha). 

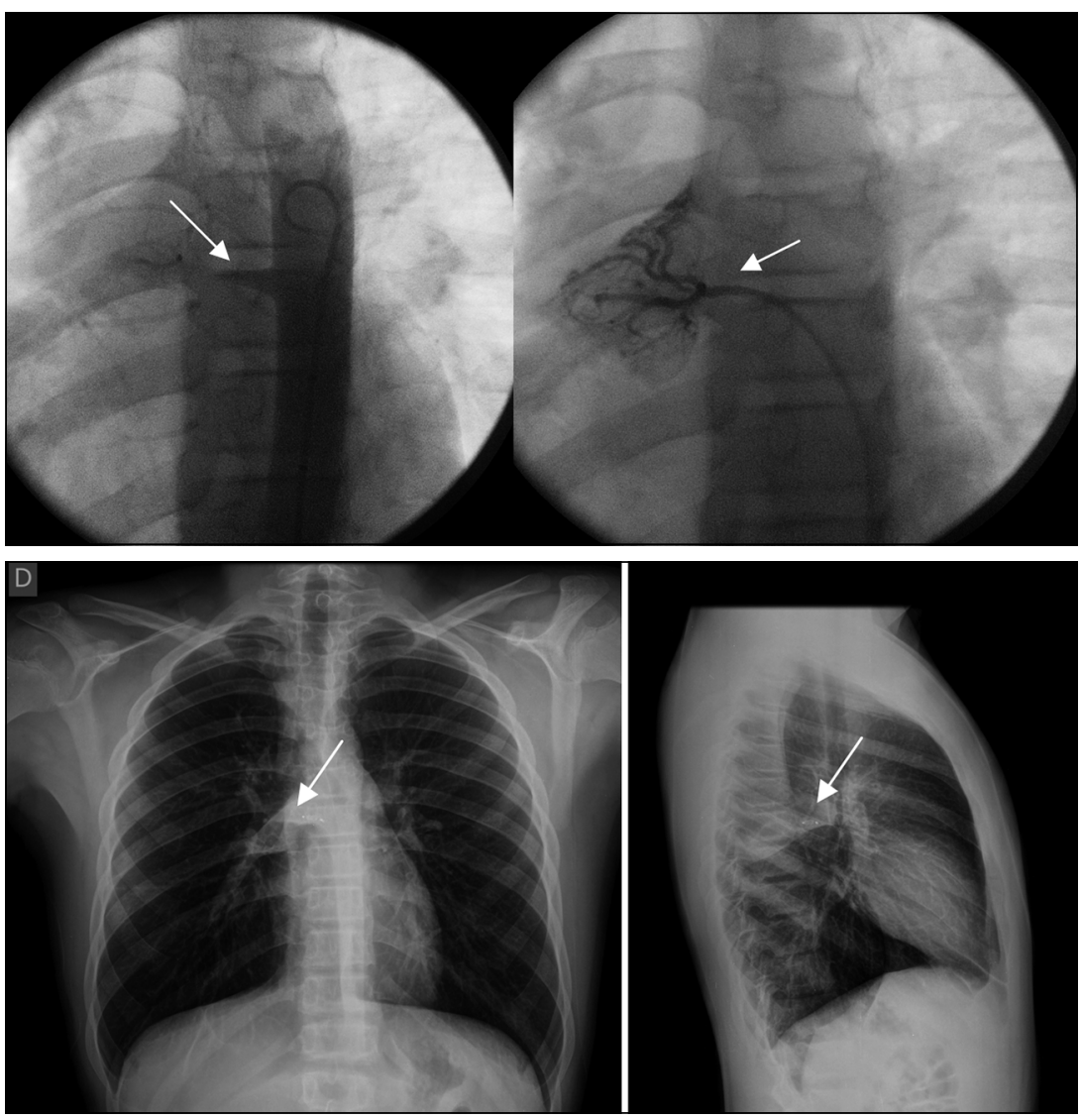

Figura 3 a y b. Aortografía a nivel torácico, la fecha indica un vaso anómalo de gran tamaño naciendo desde la aorta. En la figura 3 b se observa catéter multipropósito canulando dicho vaso y el medio de contraste muestra red capilar arteriovenosa en parénquima pulmonar del lóbulo superior derecho.

Figura 3 c y d. En la figura $3 c$ y $3 d$ se observa radiografía de torax en plano posteroanterior y lateral donde la flecha indica el dispositvo AVP II (Amplat$\operatorname{zer}^{R}$ ) en sede. de vasos que nutrían el secuestro por el cual se introdujo catéter largo de 6 French y por medio de este se implantó un dispositivo AVP II (Amplatzer $^{R}$ ) de dimensiones $4 / 6 \mathrm{~mm}$, posterior a su ubicación y comprobando su estabilidad se procedió a su liberación, obteniendo oclusión total del vaso a la angiografía de control.

El paciente fue dado de alta al día siguiente sin complicaciones y fue controlado con radiografía de tórax en la que se evidenció dispositivo in situ y campos pulmonares libres. Fue controlado a la semana, mes, a los 6 meses, al año y a los dos años, encontrándose en todos sus controles asintomático y en excelentes condiciones.

\section{Caso 3}

Recién nacido de 26 días de vida, sexo masculino que debutó con insuficiencia renal aguda e insuficiencia cardíaca de causa no precisada siendo manejado en Unidad de Cuida- dos Intensivos (UCI) con ventilación mecánica, diuréticos y drogas vasoactivas, con pobre respuesta al tratamiento. Entre los exámenes solicitados se efectuó un TAC Pulmonar con contraste que mostró un gran vaso que nacía de la aorta abdominal alta y se dirigía a la base del pulmón izquierdo planteándose el diagnóstico de SP (figura 4). Fue evaluado por cardiólogo, descartándose una cardiopatía congénita. Debido a las condiciones críticas del paciente se planificó el estudio hemodinámico y eventual embolización del vaso anómalo.

Bajo anestesia general se efectuó estudio hemodinámico, usando arteria femoral derecha con introductor 4 French se realizó una aortografía torácica con catéter Pig Tail 4 French que mostró a nivel de aorta abdominal un vaso aberrante que nacía bajo el diafragma y que se dirigía al lóbulo inferior del pulmón izquierdo con diámetro máximo de $4 \mathrm{~mm}$. Posteriormente con catéter multipropósito 4 French se cana- 


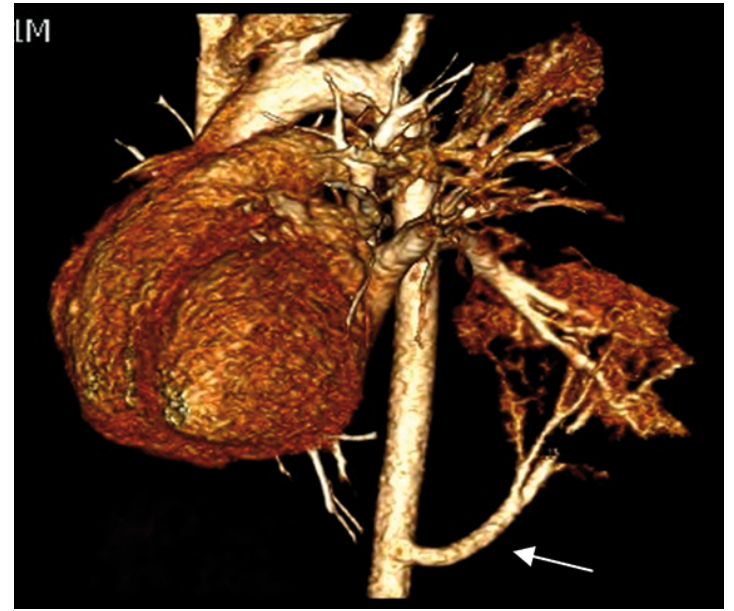

Figura 4. Imagen de reconstrucción tridimensional de AngioTAC donde se observa un gran vaso anómalo de diámetro máximo de $4 \mathrm{~mm}$ (flecha) que nace en aorta abdominal y se dirige al pulmón izquierdo para formar una red de capilares arteriovenosos en el parénquima pulmonar.

lizó el vaso y se embolizó con dispositivo coil 5PDA5 $\left(\mathrm{Cook}^{\mathrm{R}}\right)$ luego de comprobar su estabilidad se procedió a su liberación, obteniendo oclusión total del vaso a la angiografía de control después de diez minutos post implante. Procedimiento sin complicaciones.

Se controló con radiografía de tórax y ecocardiograma previo al alta evidenciando dispositivo in situ en la placa de tórax, campos pulmonares libres y cardiomegalia en franca regresión y mejora de la función cardíaca. Al día siguiente del procedimiento se logró retirar del ventilador mecánico y a las $72 \mathrm{~h}$ se suspendió el tratamiento de drogas vasoactivas, evolucionando satisfactoriamente y siendo derivado a su hospital de origen. Fue controlado a la semana, mes, a los 6 meses, al año y a los 2 años post intervención encontrándose en todos sus controles asintomático y en buenas condiciones.

En dos de los pacientes se realizó además TAC de control que mostró regresión de la lesión.

\section{Discusión}

El SP puede definirse como un segmento de pulmón con tejido ectópico no funcionante, donde no se identifica comunicación con el árbol bronquial y está irrigado por una o más ar- terias sistémicas. Si bien la mayoría de los secuestros no se comunican con las vías aéreas, ello no siempre es así. Hay dos formas de secuestro pulmonar: intrapulmonar o intralobar y extralobar o extrapulmonar, aunque pueden coexistir simultáneamente. Siendo el intralobar el más frecuente y el que presentaron nuestros pacientes. Esta patología representa 0,15$1,7 \%$ de todas las malformaciones pulmonares congénitas ${ }^{1-3}$.

La teoría de Holder y Langston establece que el secuestro intralobar es una malformación adenomatoídea congénita, o pulmón normal con una suplencia arterial aberrante que generalmente nace de la aorta. Con el tiempo, los episodios recurrentes de inflamación, pueden destruir la conexión traqueobronquial, y ocasionar la pérdida del recubrimiento celular original de la lesión. El SP se produce desde el momento en que el tejido pluripotencial del botón pulmonar adicional migra en dirección caudal con el pulmón que se está desarrollando normalmente. Éste recibe la irrigación sanguínea de los vasos que provienen de la aorta, que irrigan al intestino primitivo. Durante el período del desarrollo embrionario temprano, el botón pulmonar accesorio forma el secuestro dentro del tejido pulmonar normal encerrado en la misma pleura, constituyendo la variante intrapulmonar. En el período de desarrollo embrionario tardío, se desarrolla el botón pulmonar accesorio, dando lugar a la variante extrapulmonar de SP. La mayor parte de los casos se presenta de forma unilateral ${ }^{2,3}$, siendo rara la presentación bilateral. Nuestros pacientes tenían SP intralobar unilateral, dos afectaban el pulmón derecho y uno el izquierdo.

Para realizar el diagnóstico de SP la técnica de elección es TAC pulmonar y la angiografía por TAC que demuestra irrigación arterial sistémica desde la aorta ${ }^{4,5}$. También la Resonancia Magnética Nuclear (RMN) es de gran utilidad $^{6}$, no obstante, en nuestro medio se encuentra limitada por la falta de equipos y su alto costo. El diagnóstico antenatal se puede realizar con ultrasonido y también se ha reportado el uso de RMN fetal.

El SP puede llevar a hipertensión pulmonar, insuficiencia cardíaca congestiva por el shunt de izquierda a derecha, como uno de nuestros 
casos, que presentó insuficiencia cardíaca congestiva severa, que se complicó con insuficiencia renal y respiratoria ${ }^{1,4,5}$. En el paciente recién nacido, la sola oclusión del vaso anómalo permitió compensar la falla cardíaca y lograr retirar de ventilación mecánica.

El tratamiento, tanto en pacientes sintomáticos y asintomáticos, solía ser quirúrgico, para la extirpación del tejido no funcionante ya sea intralobar o extralobar. Este tratamiento, que generalmente se realiza por medio de una toracotomía, tiene como complicaciones la ruptura de vaso sistémico, hemorragia, infecciones, dolor y cicatriz de la zona del abordaje quirúrgico, que puede llevar deformidad músculo esquelética, asimetría del tórax, escoliosis ${ }^{7}$.

La embolización transcatéter ha sido usado como un tratamiento alternativo a la cirugía, con las ventajas de reducir el tiempo de hospitalización, no deja cicatriz, es menos invalidante y disminuye el riesgo de hemorragia ${ }^{8-11}$. Además, en niños mayores y adultos se puede efectuar sin anestesia general, tan sólo utilizando anestesia local y sedación como se efectuó en nuestros dos primeros pacientes. Es un posible tratamiento único y curativo así como se realizó en nuestra serie, logrando una mejoría de su sintomatología en el seguimiento precoz y tardío, con regresión de la lesión en los TAC de control. Para esta técnica las complicaciones reportadas incluyen dolor, fiebre, derrame pleural, isquemia transitoria de las extremidades inferiores, $y$, no se ha reportado letalidad y una baja morbilidad ${ }^{8}$. No presentándose ninguna de estas complicaciones en nuestros pacientes. El tratamiento percutáneo es también menos invalidante, no deja cicatriz y permite un alta precoz al domicilio del paciente siendo esto último muy valorado por la familia y el paciente.

\section{Conclusiones}

Existe una creciente evidencia que apoya el tratamiento con embolización transcatéter del vaso arterial anómalo que nutre al SP, como una opción segura y efectiva para tratar los secuestros pulmonares, en pacientes pediátricos. Nuestros casos se suman al pequeño pero creciente número de reportes que apoya un posible papel de esta terapia como una opción de tratamiento definitivo en los niños afectados de SP. La técnica parece ser bien tolerada y puede ayudar a evitar la morbilidad asociada con la reparación quirúrgica abierta. Esperamos que en tiempos no muy lejanos la evidencia tenga la fuerza suficiente para que esta terapéutica sea de primera elección en el tratamiento de esta patología.

\section{Referencias}

1.- Álvarez A, Borgia F, Guccione P: Regression of Left Ventricular Dilation After Percutaneous Closure of a Large Intralobar Pulmonary Sequestration Cardiology in the Young 2010; 20: 89-90.

2.- Clements BS, Warner JO: Pulmonary sequestration and related congenital bronchopulmonary vascular malformations: nomenclature and classification based on anatomical and embryological considerations. Thorax 1987; 42: 401-8.

3.- Sade RM, Clouse M, Ellis FH Jr: The spectrum of pulmonary sequestration. Ann Thorac Surg 1974; 18: 644-58.

4.- Son JS, Lee IS, Chang YP: Anomalous systemic arterial supply to normal basal segments of the right lower lobe in a neonate. Pediatr Cardiol 2008; 29: 1009-10.

5.- Wong MN, Joshi P, Sim KH: Large anomalous systemic arterial supply to the left lung without pulmonary sequestration: a rare cause of heart failure in a child. Pediatr Cardiol 2009; 30: 85-6.

6.- Tomita H, Fuse S, Chiba S: Coarctation of persistent right fifth aortic arch and pulmonary sequestration. Cardiol Young 1998; 8: 509-11.

7.- Corbett HJ, Humphrey GM: Pulmonary Sequestration. Paediatric Respiratory Reviews 2004; 5 (1): 59-68.

8.- Ganeshan A, Freedman J, Hoey ETD, Steyn R, Henderson J, Crowe PM: Transcatheter Coil Embolisation: A Novel Definitive Treatment Option for Intralobar Pulmonary Sequestration. Heart Lung and Circulation 2010; 19 (9): 561-5.

9.- Kamiyoshihara M, Nagashima T, Ichimonji I, Takeyoshi I: Three-dimensional imaging of an anomalous systemic artery supplying normal lung. Three-Dimensional Imaging of an Anomalous Systemic Artery Supplying Normal Lung. Ann Thorac Surg 2010; 90: 2060.

10.- Hwang HK, Tsai YS, Lin SM, Chen MR: Occlusion of an aberrant artery to an intralobar pulmonary sequestration using an Amplatzer Vascular Plug. Pediatr Pulmonol 2008; 43: 933-5.

11.- Tokel K, Boyvat F, Varan B: Coil embolization of pulmonary sequestration in two infants: a safe alternative to surgery. AJR Am J Roentgenol 2000; 175: 993-5. 